Cite this: RSC Advances, 2013, 3, 7828

Received 23rd December 2012, Accepted 6th March 2013

DOI: $10.1039 / c 3 r a 23454 a$

www.rsc.org/advances

\section{Chitosan fiber-supported zero-valent iron nanoparticles as a novel sorbent for sequestration of inorganic arsenic $\dagger$}

\begin{abstract}
Nesrin Horzum, a Mustafa M. Demir, ${ }^{\text {aab }}$ Muath Nairat ${ }^{c}$ and Talal Shahwan*c
This study proposes a new sorbent for the removal of inorganic arsenic from aqueous solutions. Monodispersed nano zero-valent iron (nZVI) particles were nucleated at the surface of electrospun chitosan fibers (average fiber diameter of $195 \pm 50 \mathrm{~nm}$ ) by liquid phase reduction of $\mathrm{FeCl}_{3}$ using $\mathrm{NaBH}_{4}$. The material was characterized using SEM, TGA, XPS, XRD, and FTIR. The diameter of iron nanoparticles was found to vary between $75-100 \mathrm{~nm}$. A set of batch experiments were carried out to elucidate the efficiency of the composite sorbent toward fixation of arsenite and arsenate ions. The ion concentrations in the supernatant solutions were determined using inductively coupled plasma-mass spectrometry (ICPMS). The results revealed that the chitosan fiber supported $\mathrm{nZVI}$ particles is an excellent sorbent material for inorganic arsenic uptake at concentrations ranging from 0.01 to $5.00 \mathrm{mg} \mathrm{L}^{-1}$ over a wide range of $\mathrm{pH}$ values. Based on XPS analysis, As(III) was found to undergo oxidation to As(V) upon sorption, while As(V) retained its oxidation state. By virtue of the successful combination of the electrospun fibers' mechanical integrity and the large reactivity of dispersed nZVI particles, the applicability of the resulting sorbent material in arsenic sorption holds broad promise.
\end{abstract}

\section{Introduction}

Arsenic, notoriously known for centuries as a poison, is currently considered as one of the most common and harmful water pollutants. The presence of dissolved arsenic in ground water affects the health of millions of people all around the world, and has given rise to a major environmental concern. Natural arsenic contamination of soil and surface waters mainly originates from its geological presence and oxidative weathering. The discharge of industrial wastes resulting from anthropogenic activities like burning of coal, mining-related activities, usage of fertilizers and additives in agriculture contribute to arsenic pollution in ground water. ${ }^{1}$ A primary route of exposure of human body to arsenic occurs through the consumption of contaminated water and marine food which leads to arsenic poisoning or arsenicosis. ${ }^{2}$ Long term exposure to arsenicals causes severe diseases such as keratosis, gangrene or cancer. ${ }^{3}$ Acute poisoning shows symptoms such as bloody diarrhea and vomiting. ${ }^{4}$ The predominant species of arsenic in natural ground and surface waters are either

\footnotetext{
${ }^{a}$ Department of Chemistry, Izmir Institute of Technology, Izmir, Turkey

${ }^{b}$ Department of Materials Science and Engineering, Izmir Institute of Technology,

Izmir, Turkey. E-mail: mdemir@iyte.edu.tr; Tel: +90 2327507511

${ }^{c}$ Department of Chemistry, Birzeit University, Ramallah, West Bank, Palestine.

E-mail: tshahwan@birzeit.edu; Tel: 970-2-2982146

† Electronic supplementary information (ESI) available: ICP-MS operating

parameters. See DOI: 10.1039/c3ra23454a
}

neutrally charged arsenite [As(III)] complex, or negatively charged arsenate $[\mathrm{As}(\mathrm{v})]$ oxyanions depending on $\mathrm{pH}$, redox potential (Eh), and the presence of other chemical species. Arsenic is considered by the World Health Organization (WHO) to be among the chemicals of greatest health concern in some natural waters. The maximum contamination level of arsenic in drinking water set by WHO and the US Environmental Protection Agency (EPA) is $10 \mu \mathrm{g} / \mathrm{L}$. Therefore, it is necessary to isolate arsenic from aqueous systems to avoid possible harmful exposure.

A number of arsenic removal technologies have been explored. ${ }^{4}$ Many chemical treatments include oxidation/ precipitation, ${ }^{5-8} \quad$ coagulation, ${ }^{9-14}$ sorption, ${ }^{15-24}$ ionexchange, ${ }^{25-27}$ and membrane technologies. ${ }^{28-31}$ The traditional remediation processes based on sorption are characterized by its ease of application and low-cost. ${ }^{32}$ Various sorbents are available for arsenic removal such as activated carbon, activated alumina, silica, and titanium, zirconium, iron-based materials. ${ }^{4}$ Although the efficiency of the aforementioned sorbents has been demonstrated, recent studies are proceeding for other innovative new materials. Nanoscale zero-valent iron (nZVI) technology has attracted considerable attention due to the high surface reactivity of the material which was reported to yield high arsenic sequestration ability. ${ }^{33,34}$ However, the tendency of the nanoparticles to agglomerate impedes dispersibility and stability of the material and limits their potential in water remediation. Although nZVI particles 
allow for magnetic separation techniques, ${ }^{35-38}$ their redispersion and according reusability are not readily possible due to their strong magnetic interaction. The aggregation/agglomeration of the nanoparticles reduces significantly their effective surface area, and as a result, their sorption efficiency. To overcome this problem, nZVI particles were either entrapped in a porous polymer, ${ }^{39-42}$ supported on porous materials, ${ }^{43-47}$ or nanofibrous materials. ${ }^{48-50}$ Chitosan is mostly chosen as a mechanical support because of its low-cost, wide availability, biodegradability, nontoxicity, as well as unique structural properties. The reactive amino groups on the backbone of chitosan enable fixation of several metal ions. ${ }^{51}$ However, raw chitosan is not preferred for usage in the sorption of arsenic from water because of the $\mathrm{pH}$ limitation of chitosan in the arsenic uptake, reduced accessibility of chelating groups in case of crosslinked form. ${ }^{52}$ Another disadvantage of raw chitosan is that it has poor physical nature and correspondingly low mechanical integrity leading to difficult isolation from the reaction media. ${ }^{53}$

Recently, Geng et al. proposed chitosan-stabilized nZVI particles produced by liquid phase reduction with potassium borohydride for the removal of hexavalent chromium in water. ${ }^{39}$ The usability of $\mathrm{Fe}^{0}$ nanowires modified with chitosan embedded in porous anodic alumina as sorbent towards the elimination of chromium ions was reported by Sun et $a l^{42}$ To date, chitosan-nZVI based studies have mainly focused on the remediation of chromium ions. Distinctively, Gupta et al. evaluated that nZVI encapsulated chitosan spheres are potential candidates for the adsorption of total inorganic arsenic from aqueous systems. ${ }^{40}$ Although the stability of $\mathrm{Fe}^{0}$ nanoparticles was increased by the addition of chitosan, the resulting materials still have poor physical strength which is an undesirable property for filtration processes.

In this work, we focused on the fabrication of a new functional material which allows for advantageous combination of nZVI particles and electrospun chitosan fibers. Electrospinning has been known as a versatile and efficient technique for the fabrication of fibrous materials from a wide variety of both organic and/or inorganic materials. ${ }^{54}$ Electrospun nanofibers provide excellent characteristics such as high surface area to volume ratio, enhanced surface functionalities, and superior mechanical performance compared with the powdered material. ${ }^{53}$

Due to the integrated high surface area inherent in both nanoparticles and submicron electrospun fibers which have also porous structure and high mechanical integrity, chitosan fibers-supported nZVI particles are optimal candidates for filtration applications. In this study, a facile route to fabricate a new functional material is presented. The obtained material overcomes important disadvantages such as agglomeration of the magnetic nZVI nanoparticles, and $\mathrm{pH}$ limitation of chitosan particularly in acidic environments. The material was effectively used in As(III) and As(V) uptake from aqueous systems. The main advancement for the particular material combination arises from the following issues: $(i)$ The proposed method is facile and yields a very effective material for sorption of As. (ii) The fiber material provides stable surfaces for the homogeneous dispersion of iron nanoparticles. (iii) The composite sorbent material composed of submicron sized nonwoven fibers possess high stability and mechanical integrity not only under shaking but also under continuous flow. (iv) The composite sorbent material can be repetitively used.

\section{Experimental section}

\subsection{Materials}

All the chemicals were of reagent grade and were used without any further purification. Ultrapure water (Milli-Q Millipore $18.2 \mathrm{M} \Omega \cdot \mathrm{cm}$ resistivity) was used throughout the study. Plastic containers and glassware were cleaned by soaking in diluted $\mathrm{HNO}_{3}(10 \%)$ and were rinsed with ultrapure water prior to use.

Medium molecular weight chitosan $\left(M_{\mathrm{v}}=1096 \mathrm{kDa}, 70-75 \%\right.$ degree of deacetylation, Aldrich), iron(III) chloride hexahydrate (Merck, 99\%), sodium borohydride (Aldrich, 98+\%), ethanol (Sigma-Aldrich, $\geqslant 99.5 \%$ ), $\mathrm{As}_{2} \mathrm{O}_{3}$ (Acros Organics, 99.5\%), $\mathrm{As}_{2} \mathrm{O}_{5}$ (Merck, 99\%), potassium dihydrogen phosphate (Alfa Aesar, 98\%), magnesium chloride hexahydrate (Carlo Erba, 98\%), and 1,1,1,3,3,3-hexafluoroisopropanol (HFIP, Aldrich, $\geqslant 99 \%$ ) were used as received without any further purification.

\subsection{Preparation of chitosan fiber-supported nZVI}

Scheme 1 shows a facile route for the fabrication of chitosan fiber-supported nZVI particles. In a relevant experiment, the $0.4 \mathrm{wt} \%$ chitosan solution in HFIP was transferred to a $20 \mathrm{~mL}$ plastic syringe with a needle tip. The syringe needle was connected to a high voltage supply (Gamma High Voltage Research Ormond Beach, FL, US) and a potential of $10.5 \mathrm{kV}$ was applied. The flow rate of the polymer solution was kept at $7.5 \mathrm{~mL} \mathrm{~h}^{-1}$ by using a microsyringe pump (LION WZ-50C6). The tip to collector distance was $5 \mathrm{~cm}$. When the solvent evaporated, a mat-like chitosan film was obtained on the grounded aluminum plate and stored at room temperature.

The growth of nanoparticles of zero-valent iron on the surface of the chitosan fibers was achieved by reducing iron ions using sodium borohydride. In the related experiments, $1.35 \mathrm{~g} \mathrm{FeCl}{ }_{3} \cdot 6 \mathrm{H}_{2} \mathrm{O}$ (corresponding to $0.28 \mathrm{~g}$ iron) was dissolved in $25 \mathrm{~mL}$ of $4: 1(\mathrm{v} / \mathrm{v})$ ethanol/water mixture, then $24 \mathrm{mg}$ chitosan fiber was immersed into this solution and the mixture was vigorously stirred on an orbital shaker (IKA Vortex Genius 3) for $30 \mathrm{~min} . \mathrm{Fe}^{3+}$ loaded chitosan fiber mat was then separated from the solution without any additional washing steps and placed in a beaker. In the meantime, 1.0 M sodium borohydride solutions were prepared such that $\mathrm{BH}_{4}{ }^{-}$/ $\mathrm{Fe}^{3+}$ mole ratios were $1: 1,2: 1$ and $3: 1$. The amount of $\mathrm{Fe}(\mathrm{III})$ source was kept constant, while the concentration of $\mathrm{NaBH}_{4}$ was gradually increased. The $\mathrm{NaBH}_{4}$ solution was added to chitosan fiber mat drop by drop from the burette during stirring of the reaction mixture on a magnetic stirrer. After the addition of $\mathrm{NaBH}_{4}$ solution, further mixing was maintained for $10 \mathrm{~min}$. The reduction reaction of ferric ions $\left(\mathrm{Fe}^{3+}\right)$ on chitosan fibers was marked by the formation of black particles characteristic for $\mathrm{Fe}^{0}$. The reaction can be written as: 


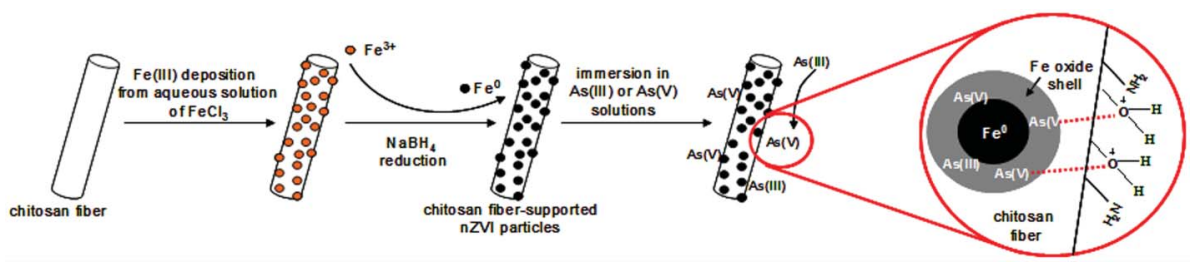

Scheme 1 Experimental steps for the preparation of chitosan fiber-supported nZVI particles and proposed mechanism of As(III)/As(V) sorption.

$$
4 \mathrm{Fe}^{3+}+3 \mathrm{BH}_{4}{ }^{-}+9 \mathrm{H}_{2} \mathrm{O} \rightarrow 4 \mathrm{Fe}^{0} \downarrow+3 \mathrm{H}_{2} \mathrm{BO}_{3}{ }^{-}+12 \mathrm{H}^{+}+6 \mathrm{H}_{2}
$$

The solid material was separated from the liquid phase by vacuum filtration. The filtrate was washed with absolute ethanol in an ultrasonic bath for $5 \mathrm{~min}$ to remove any physically adsorbed nZVI. The washing procedure was repeated three times to stabilize nZVI against immediate oxidation. Finally, the prepared material was dried at $40{ }^{\circ} \mathrm{C}$ overnight.

Scanning electron microscopy (SEM) was used for morphological characterization. This analysis was performed using a FEI Quanta250 FEG scanning electron microscope. Scanning transmission electron microscopy (STEM) mode was used to visualize nZVI particles on the surface of the chitosan nanofibers. The average fiber diameter was evaluated from the SEM micrographs for 100 individual fibers by using Fiji/ ImageJ software. SEM micrographs were used also in the estimation of the surface area coverage of the nano-zerovalent iron particles using Scandium software (Soft Imaging System Corp.). EDX elemental mappings were obtained using ZEISS Libra 200FE TEM operating at $200 \mathrm{kV}$. Small chitosan nanofibres were mounted on holey carbon film supported on copper substrate, and elemental mappings at the fibers' thin edges for iron and carbon were revealed. The thermal properties of the sorbent were analyzed using a Perkin Elmer Diamond TG/DTA.

XPS spectra were acquired for chitosan-nZVI samples before and after the removal of each $\mathrm{As}(\mathrm{III})$ and $\mathrm{As}(\mathrm{V})$ using VG Scientific ESCALAB 250 operating using $\mathrm{Al} \mathrm{K}_{\alpha}$ at $400 \mathrm{~W}$. Data analysis and evaluation were performed using CasaXPS software, the binding energies for the collected photoelectrons were referenced using C 1s peak at $284.6 \mathrm{eV}$. X-Ray diffraction (XRD) was used for structural characterization of the composite material before and after sorption of As(III) and As(V). The XRD analysis was performed using a Philips X'pert Pro diffractometer monochromatized $\mathrm{Cu} \quad \mathrm{K}_{\alpha}$ radiation $(\lambda=$ $1.540 \AA$ ). Fourier transform infrared (FTIR) spectra were acquired using a Perkin Elmer Spectrum 100 FTIR spectrometer (Shelton, U.S.A.), equipped with Pike Miracle single reflection horizontal attenuated total reflection (ATR) accessory.

\subsection{Sorption studies}

Separate stock solutions of As(III) and As(V) ions were prepared at a concentration of $1000 \mathrm{mg} \mathrm{L^{-1 }}$ by dissolving each $\mathrm{As}_{2} \mathrm{O}_{3}$ and $\mathrm{As}_{2} \mathrm{O}_{5}$ in dilute $\mathrm{HCl}$ aqueous solution $(1.0 \%, \mathrm{v} / \mathrm{v})$. The initial concentrations used in the sorption experiments were $0.01,0.05,0.10,0.25,0.50,1.00,5.00,10.0$, and $25.0 \mathrm{mg} \mathrm{L}^{-1}$, and were prepared by serial dilution from the stock solutions. The $\mathrm{pH}$ of the solutions was adjusted to 6.0 using $0.10 \mathrm{M}$ and/ or $1.0 \mathrm{M} \mathrm{NH}_{3}$ or $\mathrm{HNO}_{3}$.

Dried samples of chitosan fiber-supported zero-valent iron nanoparticles $(0.50 \mathrm{mg})$ were immersed in $3.0 \mathrm{~mL}$ synthetic As(III) and As(v) solutions and continuously shaken in a GFL 1083 water bath shaker (Burgwedel, Germany) equipped with a microprocessor. The batch sorption experiments were performed as a function of initial concentration at $25{ }^{\circ} \mathrm{C}$ for contact period of $1 \mathrm{~h}$. The eluates were then separated from the fiber sorbent by centrifugation followed by decantation. The concentrations of the metal ions in solution after sorption were determined by ICP-MS (Agilent 7500ce Series, Japan). The ICP-MS operation parameters are given in Table S1 in the Supplementary Information. $\dagger$

The effect of $\mathrm{pH}$ was studied by adjusting the initial $\mathrm{pH}$ values to 4.0, 5.0, 6.0, 7.0, 8.0 and 9.0 using $0.10 \mathrm{M}$ and/or 1.0 $\mathrm{M} \mathrm{NH}$ or $\mathrm{HNO}_{3}$. For each experiment, separate $0.50 \mathrm{mg}$ of chitosan/iron nanoparticles were placed into their respective solutions of $3.0 \mathrm{~mL}$ of $100.0 \mathrm{~g} \mathrm{~L} \mathrm{~L}^{-1} \mathrm{As}(\mathrm{III})$ and $\mathrm{As}(\mathrm{V})$. The speciation analysis of arsenic-containing ions at various $\mathrm{pH}$ values was performed using Visual MINTEQ software.

The extent of desorption was investigated by exposing the As-loaded fiber samples to $0.5 \mathrm{M} \mathrm{KH}_{2} \mathrm{PO}_{4}$ or $0.5 \mathrm{M} \mathrm{MgCl}_{2}$ for 1 $\mathrm{h}$, and were then centrifuged and the supernatant solutions were finally analyzed for possible release of As ions.

\section{Results and discussion}

\subsection{Characterization of chitosan-nZVI fibers}

The morphology and diameter distribution histogram of the chitosan fibers and chitosan fibers-supported nZVI particles are shown in Fig. 1. SEM micrograph (Fig. 1a) shows that the chitosan fibers have a smooth surface morphology with an average diameter of $195 \pm 50 \mathrm{~nm}$. ZVI nanoparticles were produced at the surface of electrospun chitosan mats by reducing $\mathrm{FeCl}_{3}$ with $\mathrm{NaBH}_{4}$. To obtain homogeneous particle dispersion on the chitosan fibers, the amount of $\mathrm{BH}_{4}{ }^{-}$with respect to $\mathrm{Fe}^{3+}$ was varied. Fig. 1b-d indicates the formation of dispersed iron nanoparticles on the surface of the chitosan fibers at different amounts of $\mathrm{BH}_{4}{ }^{-}$. At the mole ratio of $\mathrm{BH}_{4}{ }^{-}: \mathrm{Fe}^{3+}$ of $2: 1$, nZVI particles are seen to form on the fiber surface more homogeneously, with a higher density and increase in the fiber diameter compared to the mole ratio of $1: 1$. Further increases in reducing agent leads to aggregation of nZVI particles and results in separation of the particles from the fiber surface (Fig. 1d). The average fiber diameter of the 

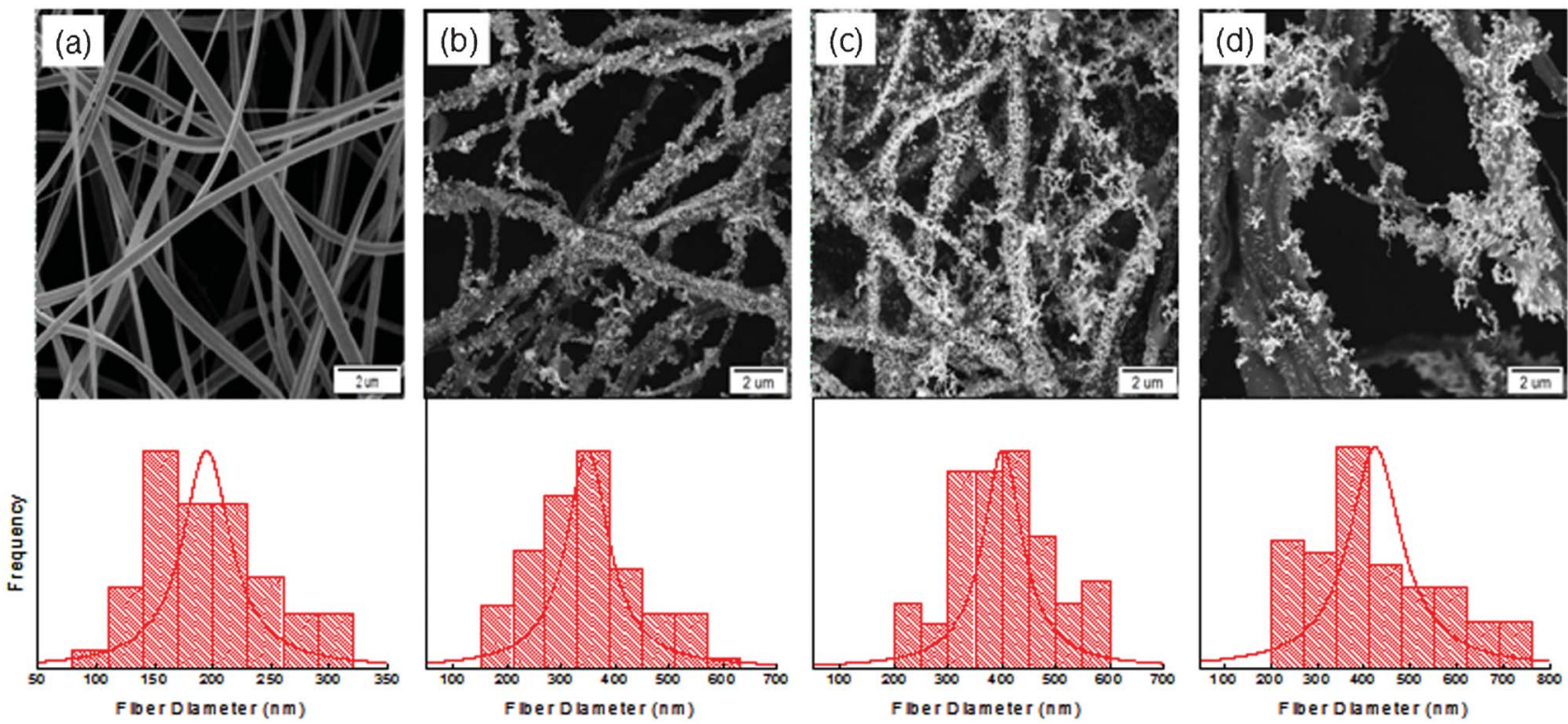

Fig. $1 \mathrm{SEM}$ micrographs and fiber diameter distributions of (a) chitosan fibers and chitosan fiber-supported nZVI particles prepared in $\mathrm{BH}_{4}{ }^{-} / \mathrm{Fe}^{3+}{ }^{3}$ atio of (b) $1: 1$ (c) $2: 1$ (d) $3: 1$.

composite fibers was determined to be $350 \pm 95 \mathrm{~nm}, 400 \pm 90$ $\mathrm{nm}$, and $425 \pm 140 \mathrm{~nm}$, respectively. The size of individual zerovalent iron nanoparticles ranged mainly between 75 and $100 \mathrm{~nm}$, although some higher scale aggregates were also observed. Fig. 2a shows an STEM micrograph of the chitosan fiber-supported nZVI particles prepared in the presence of double amounts of borohydride. Regularly shaped spherical nZVI particles appeared in dispersed form on the chitosan fibers. Typical EDX electron maps of the composite material in Fig. $2 \mathrm{~b}$ demonstrate the homogeneous distribution of $\mathrm{C}$ which forms the backbone of chitosan, while Fig. 2c reveals the presence of iron in distinct domains on the chitosan surface.

Fig. 3 displays the thermal decomposition profiles of the chitosan (curve a) and chitosan-nZVI composite fibers prepared at different $\mathrm{BH}_{4}{ }^{-} / \mathrm{Fe}^{3+}$ ratios. Curve $\mathrm{b}$ and $\mathrm{c}$ refer to $1: 1$ and $1: 2$, respectively. Both types of the fibers, regardless of the $\mathrm{BH}_{4}{ }^{-} / \mathrm{Fe}^{3+}$ mole ratio, exhibit three stages of decomposition. These are consecutive to the elimination of chemi/ physisorbed water, degradation of 2-amino-2-deoxy-D-glucopyranose, and 2-acetamido-2-deoxy-D-glucopyranose units of chitosan. ${ }^{55}$ After heating beyond $250{ }^{\circ} \mathrm{C}$, mass loss occurs

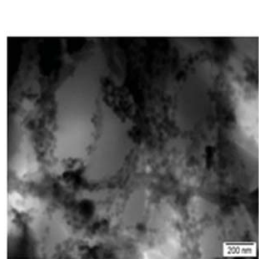

(a)

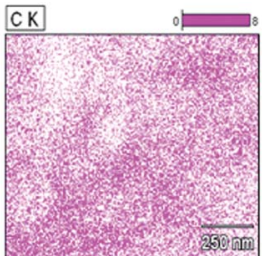

(b)

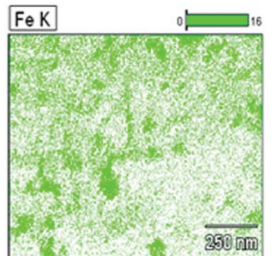

(c)
Fig. 2 (a) STEM micrograph, and elemental EDX mapping images ( $K$ lines) of the surface of chitosan fiber-supported nZVI particles (b) C, and (c) Fe. sharply and the chitosan fibers seem to be decomposed completely at temperatures higher than $600{ }^{\circ} \mathrm{C}$.

On the other hand, within the same temperature range, the composite fibers yield more than $60 \%$ of the initial mass. The remaining residue of the composite fibers can be attributed mainly to the iron based species developed on the fibers surface. Note that in comparison with the thermogram of neat chitosan fiber, the particles improve the thermal stability of chitosan-nZVI composite fibers approximately $30{ }^{\circ} \mathrm{C}$ particularly at early stage of the decomposition. Moreover, the composite fibers prepared with high $\mathrm{BH}_{4}{ }^{-} / \mathrm{Fe}^{3+}$ mole ratio yield higher amounts of inorganic residual mass (67\%) compared to the one prepared by the low ratio (57\%). The

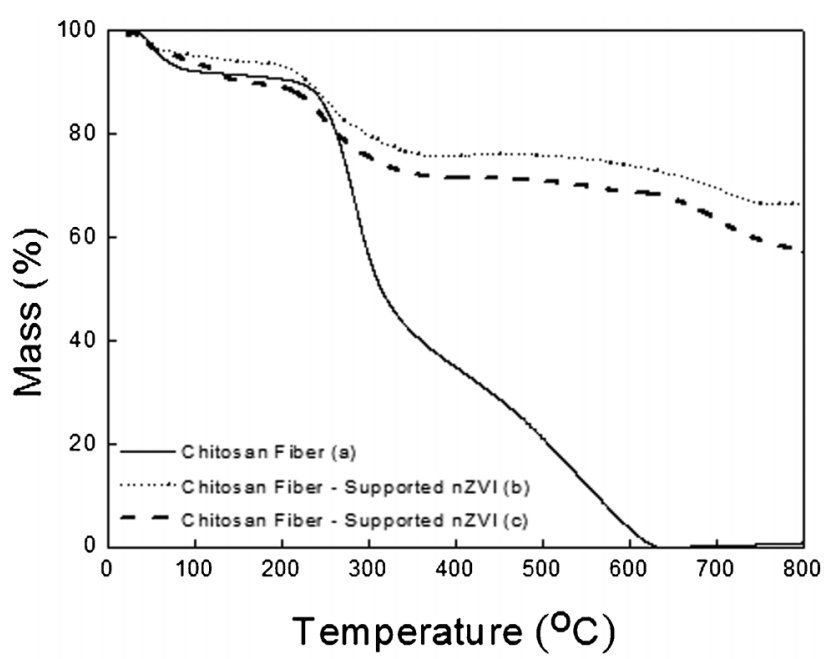

Fig. 3 TGA curves of (a) chitosan fiber, and nZVI supported on chitosan nanofibers in $\mathrm{BH}_{4}{ }^{-} / \mathrm{Fe}^{3+}$ ratio of (b) $2: 1$ (c) $1: 1$. 


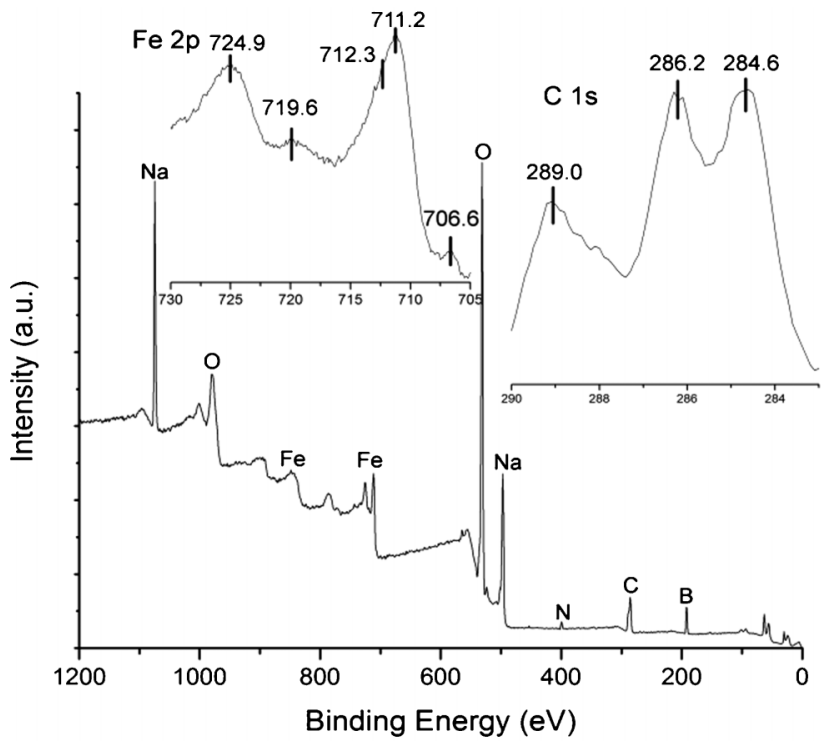

Fig. 4 Wide XPS survey of the chitosan-nZVI fibers. The insets in the figure show Fe $2 p$ and $C 1 s$ photoelectron profiles.

higher residual mass can be attributed to the formation of higher numbers of Fe-based particles developed in the presence of higher amounts of reducing agent $\left(\mathrm{BH}_{4}{ }^{-}\right)$. This result seems to be in line with the electron microscopy micrographs (see Fig. $1 \mathrm{~b}$ and c).

The chitosan-nZVI samples were analyzed using XPS. The wide survey of the sample contained signals arising from the backbone of chitosan, namely $\mathrm{C}, \mathrm{N}$, and $\mathrm{O}$, and signals that correspond to iron (Fig. 4). The iron signals are attributed mainly to the shell structure of nZVI, as the obtained signals in XPS arise from the upper few nm's of the surface. The insets in the figure correspond to $\mathrm{Fe} 2 \mathrm{p}$ and $\mathrm{C} 1 \mathrm{~s}$ photoelectron profiles. The Fe $2 p_{3 / 2}$ line appears to be centred at $711.2 \mathrm{eV}$ binding energy which corresponds to iron oxide. The shoulder that appears at $712.3 \mathrm{eV}$ is very close to binding energy values reported earlier for iron oxohydroxide $(\mathrm{FeOOH}) .{ }^{56}$ The feature at $706.6 \mathrm{eV}$ is close to the value reported for $\mathrm{Fe}$ in its zero oxidation state. ${ }^{57}$ This feature originates to the core region of the nanoparticles. The photoelectron profile of $\mathrm{C} 1 \mathrm{~s}$ shows three distinct signals that are observed at 284.6, 286.2, and $289.0 \mathrm{eV}$. These features are attributed to three different $\mathrm{C}$ environments in chitosan structure, namely $\mathrm{C}-\mathrm{C}, \mathrm{C}-\mathrm{N}$, and $\mathrm{C}-$ O environments, respectively.

The X-ray diffraction patterns of the chitosan-nZVI composite fibers before and after arsenic sorption are given in Fig. 5. The crystalline structure of nZVI is characterized by the main reflection appearing at $2 \theta$ of $44.7^{\circ}$ as shown in Fig. 5a. The absence of chitosan signals in the XRD diagram can be referred to the fact that electrospun chitosan fibers have amorphous internal structure, as reported previously elsewhere. $^{53}$

\subsection{Material properties after sorption and sorption results}

The XRD patterns of the fibers show significant changes after sorption of aqueous arsenic ions. (Fig. $5 \mathrm{~b}$ and c) The signals of

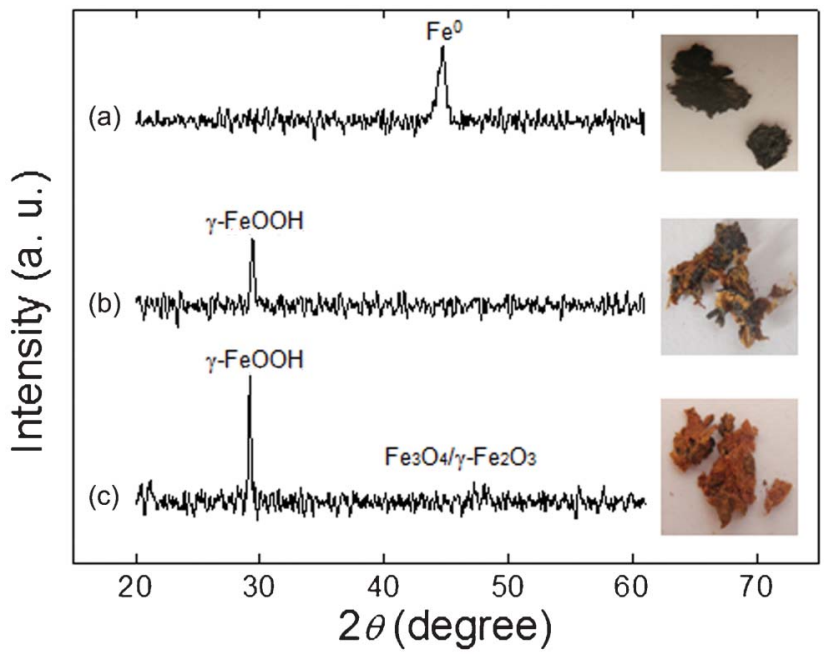

Fig. 5 XRD patterns of the chitosan fiber-supported nZVI particles (a) before sorption, (b) after As(V) sorption, (c) after As(III) sorption.

nZVI disappeared totally and the oxides appeared in the form of lepidocrocite $(\gamma$-FeOOH$)$ and magnetite/maghemite $\left(\mathrm{Fe}_{3} \mathrm{O}_{4} /\right.$ $\gamma-\mathrm{Fe}_{2} \mathrm{O}_{3}$ ). The photographic images suggest that the freshly prepared chitosan-nZVI composite fiber (Fig. 5a) is very dense and has black color. As shown in Fig. 5b and c, the fiber turns brownish orange after As(V) sorption and orange after As(III) sorption, indicating that lepidocrocite and magnetite/maghemite particles were formed on the chitosan fiber surface due to the massive oxidation during the sorption process.

The possible interactions between chitosan and nZVI particles, as well as the changes in the functional groups of chitosan-nZVI fibers after As(III) and As(V) sorption were identified by FTIR spectroscopy. Fig. 6 presents the FTIR

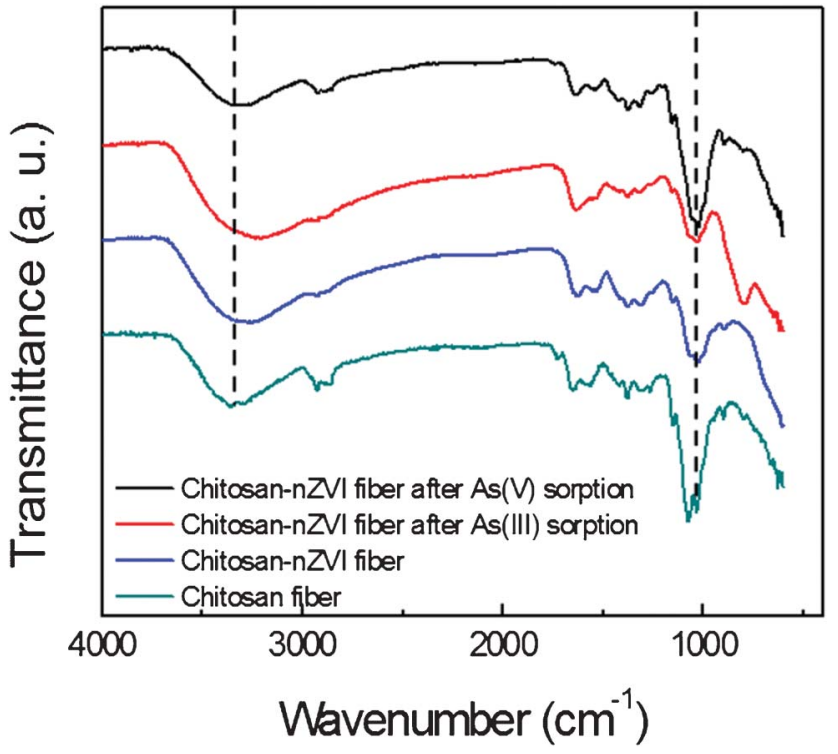

Fig. 6 FTIR spectra of the chitosan-nZVI fibers as-prepared, after As(III), and As(V) sorption, compared with the spectrum of pure chitosan fibers. 
spectra of the chitosan-nZVI fibers as-prepared, and after sorption of $\mathrm{As}(\mathrm{III})$ and $\mathrm{As}(\mathrm{V})$, together with the spectrum of neat chitosan fibers. The spectrum of the neat chitosan fibers (green curve) shows characteristic bands centred at $3332 \mathrm{~cm}^{-1}$ (O-H stretching); 2920 and $2870 \mathrm{~cm}^{-1}$ (C-H stretching); 1651 $\mathrm{cm}^{-1}$ (C-N stretching in secondary amide); $1377 \mathrm{~cm}^{-1}(\mathrm{C}-\mathrm{O}$ stretching); $1073 \mathrm{~cm}^{-1}(\mathrm{~N}-\mathrm{H}$ stretching). In the spectrum of the chitosan-nZVI fibers in Fig. 6 (blue curve), some shifts on the characteristic bands were observed depending upon the adsorption of nZVI particles on the chitosan surface. The stretching band of the hydroxyl groups at $3332 \mathrm{~cm}^{-1}$ in neat chitosan fibers are shifted to $3262 \mathrm{~cm}^{-1}$ in chitosan-nZVI fibers. On the other hand, there are shifts in the band corresponding to $\mathrm{C}-\mathrm{N}$ stretching vibrations from 1651 to 1634 $\mathrm{cm}^{-1}$, and also in the band of $\mathrm{N}-\mathrm{H}$ stretching from 1073 to $1025 \mathrm{~cm}^{-1}$. It is worth noting that the intensity of $\mathrm{C}-\mathrm{H}$, and $\mathrm{C}-$ $\mathrm{O}$ bands are significantly decreased in chitosan-nZVI fiber. The results clearly show that the interaction of nZVI with the $\mathrm{O}-\mathrm{H}$, $\mathrm{C}-\mathrm{N}$, and $\mathrm{N}-\mathrm{H}$ groups of chitosan. Such phenomenon was already reported for $\mathrm{Cu}^{58}$ and $\mathrm{Fe}^{59}$ metals, which were not only coordinated to $\mathrm{NH}_{2}$, but also formed metal complexes with $-\mathrm{OH}$ groups. To gain further insight on the sorption mechanisms, the spectra of chitosan-nZVI fibers after As(III) (red curve) and As(v) (black curve) sorption are also investigated. The stretching band of the hydroxyl groups for chitosannZVI fiber after As(III) sorption is shifted to $3206 \mathrm{~cm}^{-1}$ accompanied by an increase in the intensity. In contrast, after As(v) sorption, a blue shift is observed in the hydroxyl band with a weaker intensity. Furthermore, the intensity of $\mathrm{N}-\mathrm{H}$ stretching bands decreased in the chitosan-nZVI fiber after As(III) sorption, but increased that of the sample after As(v) sorption. The newly formed absorption band at $796 \mathrm{~cm}^{-1}$ may be attributed to the attachment of As(III) onto the chitosannZVI fibers.

The sorption experiments carried out reveal the effect of initial concentration on the sorption of $\mathrm{As}(\mathrm{III})$ and $\mathrm{As}(\mathrm{V})$ on chitosan-nZVI composite fibers, as presented in Fig. 7. These experiments were performed in the concentration range of

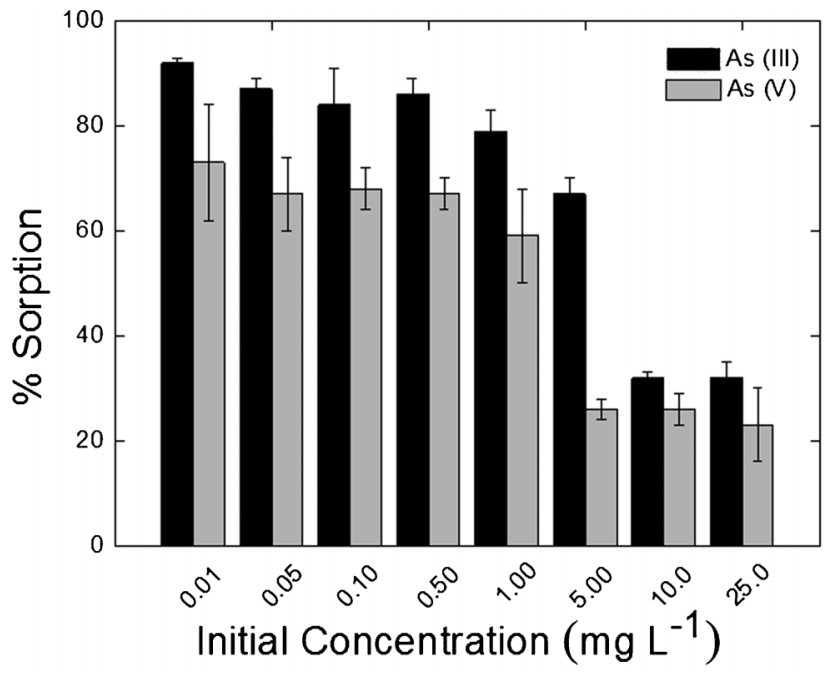

Fig. 7 Variation of \% uptake of $A s(I I I)$ and $A s(V)$ with the initial concentration.
0.01-25.0 $\mathrm{mg} \mathrm{L} \mathrm{L}^{-1}$, while the $V / M$ ratio was fixed at $60 \mathrm{~mL}$ $\mathrm{mg}^{-1}$. When the initial concentration was increased, a gradual decrease in percent sorption was observed. The data of As(III) and $\mathrm{As}(\mathrm{v})$ sorption on the chitosan-nZVI composite fiber mat were examined by Langmuir and Freundlich isotherm models, and the obtained isotherms are displayed in Fig. 8.

Langmuir theory assumes monolayer adsorption on a homogeneous surface. The non-linear form of Langmuir isotherm model is given by the equation:

$$
q_{\mathrm{e}}=q_{\max } \frac{b C_{\mathrm{e}}}{1+b C_{\mathrm{e}}}
$$

where $q_{\mathrm{e}}$, is the amount of metal ions adsorbed onto the chitosan-nZVI composite fiber sorbent $\left(\mathrm{mmol} \mathrm{g}^{-1}\right)$ at equilibrium, $C_{\mathrm{e}}\left(\mathrm{mmol} \mathrm{L}^{-1}\right)$ is the equilibrium concentration of metal ions in liquid phase, $q_{\max }\left(\mathrm{mmol} \mathrm{g}^{-1}\right)$ and $b\left(\mathrm{~L} \mathrm{mmol}^{-1}\right)$ are Langmuir constants related to the adsorption capacity and affinity, respectively.

The basic assumption of the Freundlich theory is that the adsorbent consists of a heterogeneous surface over a wide range of concentrations. Freundlich isotherm model is described by the equation:

$$
q_{\mathrm{e}}=K_{\mathrm{F}} C_{\mathrm{e}}^{1 / \mathrm{n}}
$$

Here, $K_{\mathrm{F}}$ and $n$ are the Freundlich constants for a given sorbent-sorbate system. The values of Langmuir and Freundlich constants were obtained from linear fits of the sorption data and are given in Table 1. Adequate linear correlations were observed for both Langmuir and Freundlich isotherm models. The adsorption capacities $\left(q_{\max }\right)$ of $\mathrm{As}(\mathrm{III})$ and As(v) for chitosan-nZVI composite fiber sorbent have been evaluated to be 0.0223 and $0.0305 \mathrm{mmol} \mathrm{g}^{-1}$. The Langmuir constant, $b$, can be treated as an empirical equilibrium constant, and used in the evaluation of the standard free energy of adsorption using the following equation:

$$
\Delta G^{\circ}=-R T \ln b
$$

The free energy of adsorption was found to be $-19.99 \mathrm{~kJ}$ $\mathrm{mol}^{-1}$ for As(III) and $-16.74 \mathrm{~kJ} \mathrm{~mol}^{-1}$ for As(v). The negative free energy values indicate that the adsorption reaction is driven far to the right, i.e., that products are much more favored over the reactants.

The value of Freundlich constant $(1 / n)$ reflects sorption linearity, and it usually varies between 0 and 1 . The corresponding values obtained from linear regression indicate that sorption is nonlinear, which is a typical behavior for surfaces with fixed and limited sorption capacities. The sorption of As(III) seems closer to linearity, which suggests a higher extent of sorption. The $K_{\mathrm{F}}$ values show that the fiber sorbent possesses good affinity towards the adsorbate ions, with its affinity toward As(III) being relatively higher than As(V).

The effect of solution $\mathrm{pH}$ on the uptake of As(III) and As(V) ions on the chitosan-nZVI fibers is shown in Fig. 9 at the initial concentration of $100.0 \mu \mathrm{g} \mathrm{L}^{-1}$. In the case of As(III), chitosannZVI fiber sorbent shows higher than $80 \%$ removal at $\mathrm{pH}$ within 4.0-7.0. On the other hand, a significant decrease is 

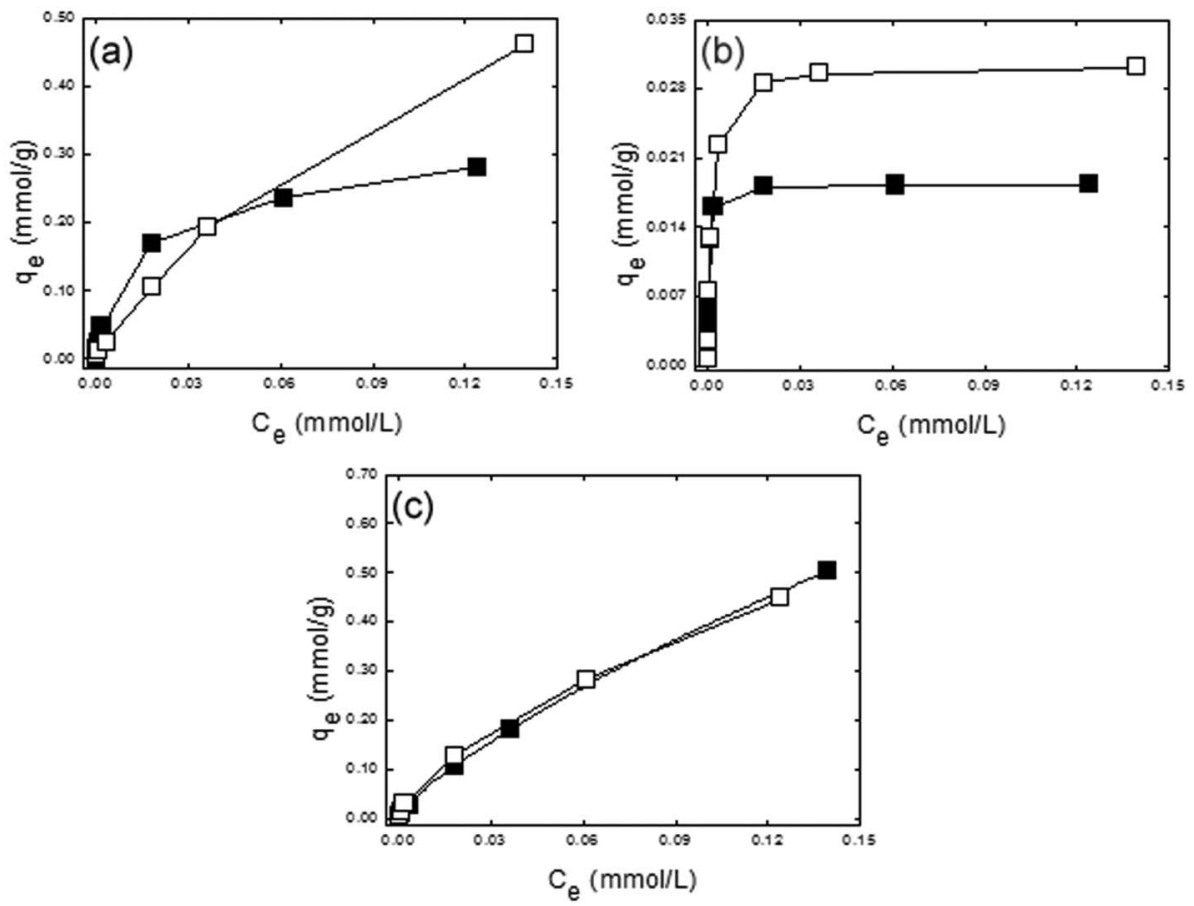

Fig. 8 (a) Adsorption isotherm and non-linear fits of (b) Langmuir, (c) Freundlich models for

As(III) and $(\square) \mathrm{As}(\mathrm{V})$, on the chitosan-nZVI composite fiber material.

observed in As(III) sorption beyond $\mathrm{pH}$ of 7.0 (Fig. 9a). A similar trend of the $\mathrm{pH}$ dependence of As(III) sorption was also observed using nano scale zero-valent iron. ${ }^{33}$ The underlying reason for the decrease in As(III) removal was explained by formation of its anionic species that undergoes repulsion by the negatively charged sorbent surface. This is confirmed by the speciation analysis provided in the inset of Fig. 9a which reveals an increase in formation of $\mathrm{H}_{2} \mathrm{AsO}_{3}{ }^{-}$in the alkaline conditions, accompanied with a decrease in the concentration of the $\mathrm{H}_{3} \mathrm{AsO}_{3}$ species.

It was reported that chitosan flakes do not show any affinity for As(III) over the wide range of $\mathrm{pH}$ values, whereas the same sorbent has the highest $\mathrm{As}(\mathrm{v})$ sorption at $\mathrm{pH} 3.0 .{ }^{60}$ However, at low $\mathrm{pH}$ values, the dissolution problem of chitosan precludes the possibility of its usage as a sorbent material for arsenic removal. As seen in Fig. 10, $\mathrm{pH}$ trials for $\mathrm{As}(\mathrm{III})$ and $\mathrm{As}(\mathrm{V})$ sorption indicate that it is also possible to remove both species at neutral $\mathrm{pH}$ values using chitosan fiber-supported nZVI

Table 1 Summary of model coefficients ${ }^{a}$

\begin{tabular}{llcc}
\hline Adsorption model & Parameter & As(III) & As(V) \\
\hline Langmuir & $R^{2}\left(\mathrm{mmol} \mathrm{g}^{-1}\right)$ & 0.9888 & 0.9958 \\
& $Q_{\max }\left(\mathrm{mmol}^{-1}\right)$ & 0.0223 & 0.0305 \\
Freundlich & $b\left(\mathrm{~L}^{2}\right.$ & 0.9950 & 856.94 \\
& $R_{\mathrm{F}}$ & 9.5499 & 0.9970 \\
& $1 / n$ & 0.8323 & 0.7601
\end{tabular}

${ }^{a}$ Solution volume, shaking time, solution $\mathrm{pH}$, sorbent amount and reaction temperature were $3.0 \mathrm{~mL}, 1 \mathrm{~h}, \mathrm{pH} 6.0,0.50 \mathrm{mg}$, and $25{ }^{\circ} \mathrm{C}$, respectively, $n=3$. particles. The nature of $\mathrm{As}(\mathrm{v})$ sorption around $\mathrm{pH} 4-8$, can be explained by coulombic interaction between chitosan-nZVI surfaces which exhibit a positive charge at $\mathrm{pH}$ lower than the point of zero charge of 7.8 for $\mathrm{nZVI}^{33}$ and $\mathrm{H}_{2} \mathrm{AsO}_{4}{ }^{-} / \mathrm{HAsO}_{4}{ }^{2-}$ ions which are the main species at the $\mathrm{pH}$ range.

It is worth noting that the fate of iron was taken into account while removing the arsenicals by using chitosan-nZVI

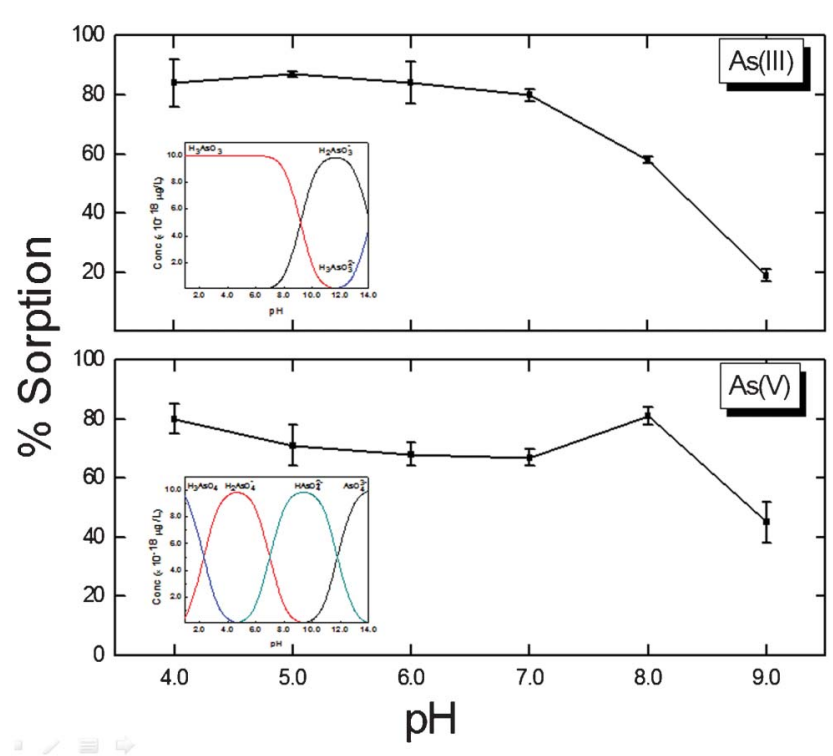

Fig. 9 Effect of pH on the sorption of $100.0 \mu \mathrm{L} \mathrm{L}^{-1}$ (a) $\mathrm{As}(\mathrm{III})$, and (b) $\mathrm{As}(\mathrm{V})$, solutions by chitosan-nZVI fibers. The insets show speciation diagrams of As(III) and $\operatorname{As}(V)$ 

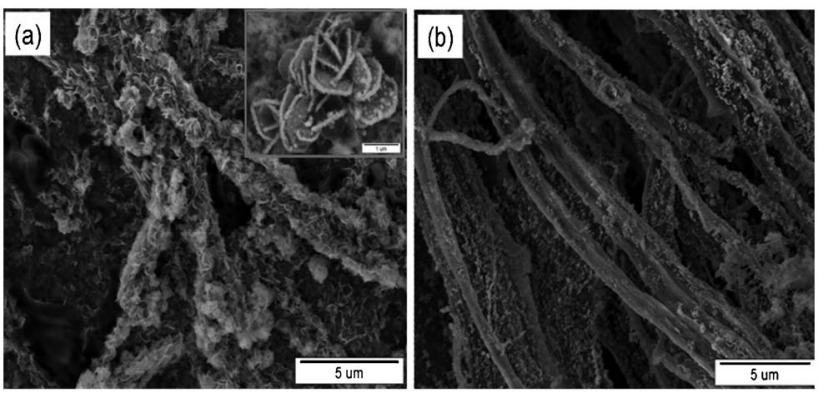

Fig. 10 SEM micrographs of the chitosan-nZVI composite fiber after (a) As(III), and (b) As(v) sorption.

fibers. Higher amounts of dissolved iron were determined after As(v) sorption compared with the iron content after As(III) sorption. The maximum iron concentration in solution after the sorption process was $14 \mathrm{mg} \mathrm{L}^{-1}$. It is estimated that the amount of dissolved iron is less than $0.7 \%$ of the initial nZVI amount assuming that the surface of chitosan is entirely coated with the particles.

Desorption experiments were performed in an analogous way to those with sorption experiments. The arsenic ionloaded chitosan-nZVI fiber sorbents were individually treated with $0.5 \mathrm{M} \mathrm{MgCl}_{2}$ or $\mathrm{KH}_{2} \mathrm{PO}_{4}$ for the release of arsenic ions. The desorption percentages for As(III) ions were $49 \%$ for $\mathrm{KH}_{2} \mathrm{PO}_{4}, 40 \%$ for $\mathrm{MgCl}_{2}$, and for $\mathrm{As}(\mathrm{v})$ ions were $36 \%$ for $\mathrm{KH}_{2} \mathrm{PO}_{4}, 24 \%$ for $\mathrm{MgCl}_{2}$. This indicates that the sorbed $\mathrm{As}(\mathrm{v})$ species are more stable than those of As(III).

SEM micrographs of dried chitosan-nZVI composite fibers after As(III) (Fig. 10a) and As(V) (Fig. 10b) sorption studies showed a bundled fibrous structure along with lepidocrocite and/or magnetite/maghemite particles on the electrospun chitosan fibers. Agglomeration of iron oxide particles is also demonstrated by the figures. Distinctively, flower like structures on/between the fibers (Fig. 10a, inset) appeared after As(III) sorption.

The XPS features of $\mathrm{Fe} 2 \mathrm{p}$ before and after sorption of arsenic species are shown in Fig. 11A. The oxidation of Fe is marked by the disappearance of $\mathrm{Fe}^{0}$ feature that appears in curve a of Fig. 11A around $707 \mathrm{eV}$. The oxidation leads to a shift in the Fe $2 \mathrm{p}$ peaks towards higher binding energies, indicating the development of iron oxyhydroxide groups on the surface. This is seen to be more pronounced in the case of As(v) sorption (curve $\mathrm{c}$ in Fig. 11A), possibly leading to circumstances in which the ion is liable to complexation by oxyhydroxyl groups.

The $\mathrm{O}$ 1s profiles corresponding to the samples before and after sorption are given in Fig. 11B. The peak envelope centred around 531.0-531.3 eV showed relatively little changes, indicating that it belongs to structural $\mathrm{O}$ in the composite materials. The variety of chemical environments in which $\mathrm{O}$ exists in the composite materials makes it difficult to provide an accurate assignment for these envelopes of peaks. Nevertheless, it is possible to mention that these features display binding energies close to those assigned earlier for $\mathrm{O}^{2-}$ in the hydroxyl groups of $\alpha-\mathrm{FeOOH}$ (goethite) and $\gamma$-FeOOH (lepidocrocite) reported at $531.0 \mathrm{eV}$ and $531.1 \mathrm{eV}$, respec-
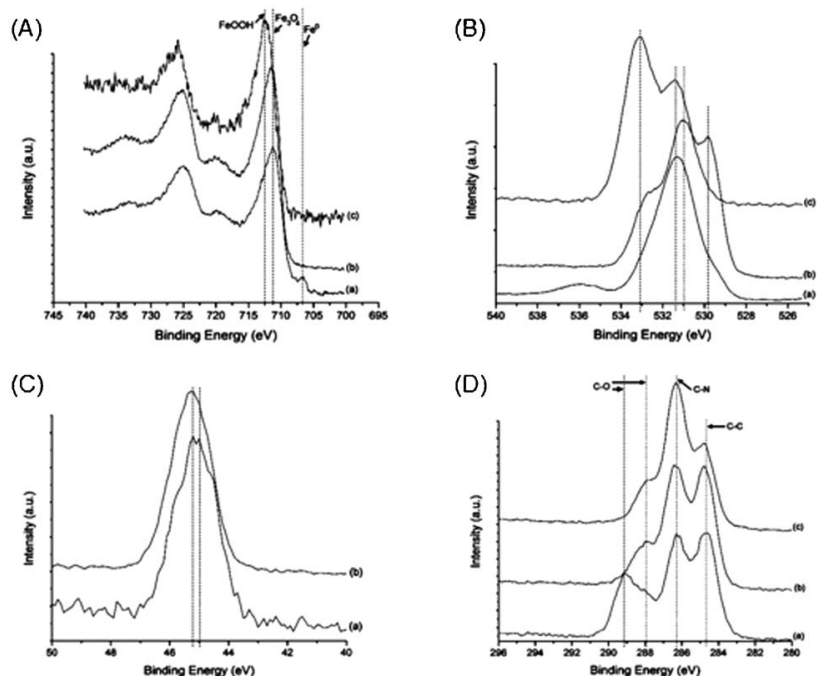

Fig. 11 XPS profiles of the composite fibers showing (A) Fe $2 p$, (B) O 1s, (C) As 3d, (D) C 1s features before and after sorption process. In Fig. A, B, D; (a) represents the samples before sorption, (b) refers the samples after sorption of $\mathrm{As}(\mathrm{III})$, and (c) the samples after sorption of As(V). In Fig. C, (a) stands for As(III) signal and (b) stands for As(V) signal.

tively. ${ }^{61}$ The sorption of As(III) leads to the development of an $\mathrm{O} 1 \mathrm{~s}$ feature centred at $529.8 \mathrm{eV}$ and a shoulder centred at $533.1 \mathrm{eV}$ (curve b in Fig. 11B). The former one $(529.8 \mathrm{eV})$ is close to those assigned previously to $\mathrm{O}^{2-}$ in $\alpha-\mathrm{Fe}_{2} \mathrm{O}_{3}$ (hematite) and that in $\gamma-\mathrm{Fe}_{2} \mathrm{O}_{3}$ (maghemite), reported at $530.0 \mathrm{eV}$ and $529.9 \mathrm{eV}$, respectively, ${ }^{61}$ and could reflect the extensive oxidation of iron accompanying sorption. The latter feature at $533.1 \mathrm{eV}$ is tentatively thought to belong to the $\mathrm{O}$ in the sorbed arsenic species. This feature becomes more distinct when $\mathrm{As}(\mathrm{V})$ is sorbed (curve c in Fig. 11B). Given that $\mathrm{As}(\mathrm{III})$ is largely oxidized to As(V) upon sorption, as discussed in the next section, and the fact that $\mathrm{O}$ 1s level bonded to As(v) would experience a decrease in electron density, it seems logical to assign this feature to sorbed arsenate species.

\subsection{Sorption mechanism}

The sorption mechanism of As species by nZVI has been addressed in several studies, and seems not to be totally resolved yet. Earlier studies reported that As(III) undergoes fast sorption on nZVI surface, and as time passes, As(III) near or in contact with the corroding nZVI surface may be oxidized to $\mathrm{As}(\mathrm{v})$, which subsequently is sorbed by an inner-sphere mechanism. ${ }^{33}$ In a study of As(v) sorption on nZVI, it was confirmed that As(v) is fixed through an inner-sphere surface complexation. ${ }^{34}$ The same study indicated that until 60 days, only As(v) was detected, but on a longer time scale As(III) was identified due to the reduction of $\mathrm{As}(\mathrm{V})$.

In later studies, it was shown that As(III) undergoes both oxidation and reduction at nZVI particle surfaces. ${ }^{62}$ Oxidation was reported to yield As(v), while reduction lead to formation of As(0). This was confirmed in another study in which it was stated that $\operatorname{As}(\mathrm{V})$, formed via oxidation of As(III), was fixed predominantly by outer surface of the oxide shell of nZVI surface, while reduced species were enriched in a subsurface 
layer close to the $\mathrm{Fe}(0)$ core $^{63}$ Recently, it was reported, based on analysis using the structural techniques X-ray absorption spectroscopy (XAS) and X-ray absorption near-edge spectroscopy (XANES) that for nZVI in anoxic As(III) solutions, As(III) species sorbed on nZVI surface undergoes diffusion across the thin oxide layer enclosing the nanoparticles, which results in arsenic forming an intermetallic phase with the $\mathrm{Fe}(0)$ core. $^{64}$ The same study reported that As(v), presumably resulting from As(III), could not be detected, and attributed its detection in other studies to alteration of surface chemistry of nZVI during XPS analysis.

The XPS profiles obtained in this study for the sorption of As(III) and As(V) are shown in Fig. 11C. The XPS profile of As 3d resulting from the sorption of $\mathrm{As}(\mathrm{V})$ seems to be centred at 45.2 eV (curve b in Fig. 11C). Similarly, the profile of As 3d resulting from the sorption of As(III) is slightly split to two peaks centred at $45.2 \mathrm{eV}$ and $45.0 \mathrm{eV}$ (curve a in Fig. 11C). Based on this, it seems that, while As(v) retains its oxidation state upon fixation by nZVI surface, As(III) undergoes oxidation to As(v). The split might suggest the formation of two types of surface complexes of As(v). The absence of a signal at $43.5 \mathrm{eV}$ indicates that As(III) is totally oxidized. Moreover, no signal of $\mathrm{As}(0)$ was observed in both cases, suggesting that reduction is not involved in the mechanism. These results should be viewed within the context of conditions applied in this study. The formation of As(0) was well documented before as one of the options of the fixation mechanisms for both As(III) and As(v) on nZVI. ${ }^{62}$ However, the same study mentioned arsenic concentration and solution $\mathrm{pH}$, and the properties of iron materials play crucial roles in determining the final arsenic speciation.

The contribution of chitosan component in the composite material to the uptake of As ions cannot be ruled out. This has been well documented in literature, ${ }^{65}$ and is obvious from the changes observed in the relative intensities of $\mathrm{C} 1 \mathrm{~s}$ components in the XPS spectrum. It is important to mention that the fixation of As(III) and As(V) resulted in a great decrease in the intensity and shift in the binding energy of $\mathrm{C} 1 \mathrm{~s}$ feature corresponding to $\mathrm{C}-\mathrm{O}$ environment (Fig. 11D), possibly indicating that the hydroxyl groups of chitosan form more attractive centers for arsenite and arsenate complexation. In both cases, no important change in the $\mathrm{C}$ 1s feature corresponding to $\mathrm{C}-\mathrm{N}$ environment is observed. It could be useful to note also that the sorption of $\mathrm{As}(\mathrm{v})$ led to a large decrease in the $\mathrm{C} 1 \mathrm{~s}$ intensity corresponding $\mathrm{C}-\mathrm{C}$ environment, possibly suggesting partial disruption of these bonds in chitosan structure upon sorption of As(v) species. As revealed by the speciation analysis mentioned above, within the $\mathrm{pH}$ conditions used of this study, the major forms of As(III) are expected to be $\mathrm{H}_{3} \mathrm{AsO}_{3} / \mathrm{H}_{2} \mathrm{AsO}_{3}{ }^{-}$, while the major forms of $\mathrm{As}(\mathrm{V})$ are $\mathrm{H}_{2} \mathrm{AsO}_{4}{ }^{-} / \mathrm{HAsO}_{4}{ }^{2-}$, hence the mode of interaction between these species and the protonated hydroxyl groups on chitosan surface would be dominated by electrostatic attraction. Due to its neutral $\mathrm{H}_{3} \mathrm{AsO}_{3}$ form, the extent of sorption of As(III) by chitosan component is expected to be smaller than that of $\operatorname{As}(\mathrm{v})$.

\section{Conclusions}

Chitosan fiber-supported nZVI particles were synthesized and then used in the removal of inorganic arsenic species from aqueous systems. High sorption capacity and stable performance of the chitosan-nZVI fibers were achieved over a wide range of $\mathrm{pH}$. The fiber sorbent showed higher affinity toward $\mathrm{As}(\mathrm{III})$ compared to $\mathrm{As}(\mathrm{V})$, and the sorption behavior was adequately described using both Langmuir and Freundlich isotherms. Spectroscopic investigation revealed that the arsenic ions seem to be fixed to oxide and oxyhydroxide groups at the outer shells of nZVI surfaces. While As(v) retains its oxidation state upon fixation by nZVI, As(III) undergoes oxidation to As(v). Moreover, chitosan seems to contribute to arsenite and arsenate complexation mainly via its hydroxyl groups.

The ease of preparation of chitosan fiber-supported nZVI particles can offer a very convenient way for the development of sorption-enhanced materials to use in the removal of undesirable metal ions from water.

\section{Acknowledgements}

The authors express their gratitude for Mr. A. Timmer, Dr. A. Schäfer and Dr. H. Rösner at the Center for Nanotechnology and the Physics Institute in Münster, Germany, for their help in XPS and EDX mapping analysis. The authors acknowledge the Center of Material Research and the Environmental Research Center at IzTech for the rest of the measurements.

\section{Notes and references}

1 B. K. Mandal and K. T. Suzuki, Talanta, 2002, 58, 201.

2 J. C. Saha, A. K. Dikshit, M. Bandyopadhyay and K. C. Saha, Crit. Rev. Environ. Sci. Technol., 1999, 29, 281.

3 T. S. Y. Choong, T. G. Chuah, Y. Robiah, F. L. G. Koay and I. Azni, Desalination, 2007, 217, 139.

4 D. Mohan and C. U. Pittman, J. Hazard. Mater., 2007, 142, 1.

5 L. H. Sun, R. P. Liu, S. J. Xia, Y. L. Yang and G. B. Li, Desalination, 2009, 243, 122.

6 O. X. Leupin and S. J. Hug, Water Res., 2005, 39, 1729.

7 M. Bissen and F. H. Frimmel, Acta Hydrochim. Hydrobiol., 2003, 31, 97.

8 T. M. Gihring, G. K. Druschel, R. B. McCleskey, R. J. Hamers and J. F. Banfield, Environ. Sci. Technol., 2001, 35, 3857.

9 E. Lacasa, P. Canizares, C. Saez, F. J. Fernandez and M. A. Rodrigo, Sep. Purif. Technol., 2011, 79, 15.

10 D. Lakshmanan, D. A. Clifford and G. Samanta, Water Res., 2010, 44, 5641.

11 N. Balasubramanian, T. Kojima and C. Srinivasakannan, Chem. Eng. J., 2009, 155, 76.

12 D. Lakshmanan, D. Clifford and G. Samanta, J. Am. Water Work Assoc., 2008, 100, 76. 
13 S. Song, A. Lopez-Valdivieso, D. J. Hernandez-Campos, C. Peng, M. G. Monroy-Fernandez and I. Razo-Soto, Water Res., 2006, 40, 364.

14 S. R. Wickramasinghe, B. B. Han, J. Zimbron, Z. Shen and M. N. Karim, Desalination, 2004, 169, 231.

15 P. Chutia, S. Kato, T. Kojima and S. Satokawa, J. Hazard. Mater., 2009, 162, 204.

16 V. M. Boddu, K. Abburi, J. L. Talbott, E. D. Smith and R. Haasch, Water Res., 2008, 42, 633.

17 J. Gimenez, M. Martinez, J. de Pablo, M. Rovira and L. Duro, J. Hazard. Mater., 2007, 141, 575.

18 C. C. Chen and Y. C. Chung, J. Environ. Sci. Health Part A-Toxic/Hazard. Subst. Environ. Eng., 2006, 41, 645.

19 E. Deschamps, V. S. T. Ciminelli and W. H. Holl, Water Res., 2005, 39, 5212.

20 L. Cumbal and A. K. Sengupta, Environ. Sci. Technol., 2005, 39, 6508 .

21 B. Dousova, V. Machovic, D. Kolousek, F. Kovanda and V. Dornicak, Water, Air, Soil Pollut., 2003, 149, 251.

22 M. J. DeMarco, A. K. Sengupta and J. E. Greenleaf, Water Res., 2003, 37, 164.

23 V. Lenoble, O. Bouras, V. Deluchat, B. Serpaud and J. C. Bollinger, J. Colloid Interface Sci., 2002, 255, 52.

24 L. Dambies, E. Guibal and A. Roze, Colloids Surf., A, 2000, 170, 19.

25 B. An, T. R. Steinwinder and D. Y. Zhao, Water Res., 2005, 39, 4993.

26 J. Kim and M. M. Benjamin, Water Res., 2004, 38, 2053.

27 G. L. Ghurye, D. A. Clifford and A. R. Tripp, J. Am. Water Work Assoc., 1999, 91, 85.

28 A. E. Pagana, S. D. Sklari, E. S. Kikkinides and V. T. Zaspalis, Microporous Mesoporous Mater., 2008, 110, 150.

29 J. Iqbal, H. J. Kim, J. S. Yang, K. Baek and J. W. Yang, Chemosphere, 2007, 66, 970.

30 M. C. Shih, Desalination, 2005, 172, 85.

31 R. Y. Ning, Desalination, 2002, 143, 237.

32 B. Daus, R. Wennrich and H. Weiss, Water Res., 2004, 38, 2948.

33 S. R. Kanel, B. Manning, L. Charlet and H. Choi, Environ. Sci. Technol., 2005, 39, 1291.

34 S. R. Kanel, J. M. Greneche and H. Choi, Environ. Sci. Technol., 2006, 40, 2045.

35 Z. H. Ai, Y. Cheng, L. Z. Zhang and J. R. Qiu, Environ. Sci. Technol., 2008, 42, 6955.

36 A. E. Karatapanis, D. E. Petrakis and C. D. Stalikas, Anal. Chim. Acta, 2012, 726, 22.

37 X. Q. Li, D. W. Elliott and W. X. Zhang, Crit. Rev. Solid State Mater. Sci., 2006, 31, 111.

38 Y. C. Sharma, V. Srivastava, V. K. Singh, S. N. Kaul and C. H. Weng, Environ. Technol., 2009, 30, 583.

39 B. Geng, Z. H. Jin, T. L. Li and X. H. Qi, Sci. Total Environ., 2009, 407, 4994.
40 A. Gupta, M. Yunus and N. Sankararamakrishnan, Chemosphere, 2012, 86, 150.

41 T. Y. Liu, L. Zhao and Z. L. Wang, Water Sci. Technol., 2012, 66, 1044.

42 L. Sun, L. D. Zhang, C. H. Liang, Z. G. Yuan, Y. Zhang, W. Xu, J. X. Zhang and Y. Z. Chen, J. Mater. Chem., 2011, 21, 5877.

43 L. N. Shi, X. Zhang and Z. L. Chen, Water Res., 2011, 45, 886.

44 D. Karabelli, S. Unal, T. Shahwan and A. E. Eroglu, Chem. Eng. J., 2011, 168, 979.

45 T. Shahwan, C. Uzum, A. E. Eroglu and I. Lieberwirth, Appl. Clay Sci., 2010, 47, 257.

46 C. Uzum, T. Shahwan, A. E. Eroglu, K. R. Hallam, T. B. Scott and I. Lieberwirth, Appl. Clay Sci., 2009, 43, 172.

47 S. L. Xiao, M. W. Shen, R. Guo, S. Y. Wang and X. Y. Shi, J. Phys. Chem. C, 2009, 113, 18062.

48 S. L. Xiao, M. W. Shen, R. Guo, Q. G. Huang, S. Y. Wang and X. Y. Shi, J. Mater. Chem., 2010, 20, 5700.

49 S. L. Xiao, H. Ma, M. W. Shen, S. Y. Wang, Q. G. Huang and X. Y. Shi, Colloids Surf., A, 2011, 381, 48.

50 H. Ma, Y. P. Huang, M. W. Shen, R. Guo, X. Y. Cao and X. Y. Shi, J. Hazard. Mater., 2012, 211, 349.

51 S. E. Bailey, T. J. Olin, R. M. Bricka and D. D. Adrian, Water Res., 1999, 33, 2469.

52 G. Crini, Prog. Polym. Sci., 2005, 30, 38.

53 N. Horzum, E. Boyaci, A. E. Eroglu, T. Shahwan and M. M. Demir, Biomacromolecules, 2010, 11, 3301.

54 Z. M. Huang, Y. Z. Zhang, M. Kotaki and S. Ramakrishna, Compos. Sci. Technol., 2003, 63, 2223.

55 Y. S. Nam, W. H. Park, D. Ihm and S. M. Hudson, Carbohydr. Polym., 2010, 80, 291.

56 C. D. Wagner, W. M. Riggs, L. E. Davis, J. F. Moulder and G. E. Muilenberg, Perkin-Elmer Corporation, Eden Prairie, Minnesota, 1979.

57 M. Oku and K. Hirokawa, J. Appl. Phys., 1979, 50, 6303.

58 O. A. C. Monteiro and C. Airoldi, J. Colloid Interface Sci., 1999, 212, 212.

59 S. C. Bhatia and N. Ravi, Biomacromolecules, 2003, 4, 723.

60 E. Boyaci, A. E. Eroglu and T. Shahwan, Talanta, 2010, 80, 1452.

61 A. P. Grosvenor, B. A. Kobe, M. C. Biesinger and N. S. McIntyre, Surf. Interface Anal., 2004, 36, 1564.

62 M. A. V. Ramos, W. Yan, X. Q. Li, B. E. Koel and W. X. Zhang, J. Phys. Chem. C, 2009, 113, 14591.

63 W. L. Yan, M. A. V. Ramos, B. E. Koel and W. X. Zhang, Chem. Commun., 2010, 46, 6995.

64 W. Yan, R. Vasic, A. I. Frenkel and B. E. Koel, Environ. Sci. Technol., 2012, 46, 7018.

65 L. Pontoni and M. Fabbricino, Carbohydr. Res., 2012, 356, 86. 